\title{
Nonlinear orthotropic model of the inhomogeneous assembly compression of PEM fuel cell gas diffusion layers
}

\author{
Pablo A. García-Salaberri ${ }^{a}$, Marcos Vera $^{a, *}$, Ramón Zaera ${ }^{b}$ \\ ${ }^{a}$ Departamento de Ingeniería Térmica y de Fluidos, Universidad Carlos III de Madrid, Auda. de la Universidad, 30,28911 Leganés, Spain \\ b Departamento de Mecánica de Medios Continuos y Teoría de Estructuras, Universidad Carlos III de Madrid, 28911 Leganés, Spain
}

\section{Keywords:}

PEM fuel cells

Assembly pressure

Gas diffusion layers

Inhomogeneous compression

Nonlinear orthotropic model

\begin{abstract}
A B S T R A C T
PEM fuel cell assembly pressure is known to cause large strains in the gas diffusion layer (GDL), which results in significant changes in its mechanical, electrical and thermal properties. These changes affect the rates of mass, charge, and heat transport through the GDL, thus impacting fuel cell performance and lifetime. The appropriate modeling of the inhomogeneous GDL compression process associated with the repetitive channel rib pattern is therefore essential for a detailed description of the physical chemical processes that take place in the cell. In this context, the mechanical characterization of the GDL is of special relevance, since its microstructure based on carbon fibers has strongly nonlinear orthotropic properties. The present study describes a new finite element model which fully incorporates the nonlinear orthotropic characteristics of the GDL, thereby improving the prediction of the inhomogeneous compression effects in this key element of the cell. Among other conclusions, the numerical results show that the linear isotropic models widely reported in the literature tend to overestimate the porosity and the partial intrusion of the GDL in the channel region, and may lead to incorrect predictions in terms of interfacial contact pressure distributions.
\end{abstract}

\section{Introduction}

PEM fuel cells are electrochemical devices that convert the chemical energy of an energy carrier and an oxidizer-typically oxygen-directly into electricity and heat [1]. Depending on whether they use hydrogen or methanol as energy carrier, they are commonly known as proton exchange membrane fuel cells (PEMFCs) or direct methanol fuel cells (DMFCs), respectively. In both cases, the electrochemical reactions are facilitated by the presence of a noble metal catalyst, so that no conventional high temperature combustion processes occur in the cell. This approach substantially reduces the emissions of air pollutants and results in a more energetically efficient process due to the lack of any thermodynamic efficiency limit. Further benefits of PEM fuel cells include low noise emissions, modular and pro longed operation, high reliability, fast response, and short recharge times. PEM fuel cells are therefore regarded as poten tial substitutes to conventional power sources for stationary, portable, and automotive applications [2].

The core component of a PEM fuel cell consists of a five layered structure called the membrane electrode assembly (MEA), which is formed by a polymer electrolyte membrane (PEM) with a thin layer of catalyst on both sides, and a porous gas diffusion layer (GDL) in contact with each of the catalyst layers [3]. The MEA is further sandwiched between the bipolar plates, which supply reactants to and remove products (and

\footnotetext{
* Corresponding author. Tel.: +34 91624 9987; fax: +34 916249430.

E mail address: marcos.vera@uc3m.es (M. Vera).
} 
heat) from the active areas of the cell, act as current collectors, and provide mechanical support for the cells in the stack $[4,5]$.

One of the key elements affecting PEM fuel cell performance is the GDL, which must provide a passage for reactant access and excess product removal to/from the catalyst layers, high electronic and thermal conductivity, and adequate mechanical support for the MEA. In order to fulfill these requirements, GDLs are typically made of highly porous carbon fiber paper or cloth $[6,7]$. The high porosity of these materials provides to the GDL a characteristic soft and flexible structure, susceptible of large deformations when subjected to compression. This leads to significant changes in its mechanical, electrical and thermal properties (thickness, porosity, permeability, electrical and thermal bulk conductivities and contact resistances, etc.), thus affecting mass, charge, and heat transfer processes, fuel cell performance and lifetime [8-15].

GDL compression occurs during the assembly/disassembly process of the stack [16,17], but also during fuel cell operation due to membrane swelling (an effect that is not considered in this paper) [18-24]. Additionally, the repetitive channel rib pattern of the bipolar plates results in a highly inhomoge neous compressive load, so that while large strains are produced under the rib, typically between 10 and $40 \%$ of the initial thickness [28-30], the region under the channels remains approximately at its initial uncompressed state. This leads to significant spatial variations in GDL thickness and porosity distributions, as well as in electrical [25,26] and thermal [27] bulk conductivities and contact resistances (both at the rib-GDL and membrane-GDL interfaces).

The inhomogeneous compression of the GDL leads to several opposing effects. On one hand, the assembly pressure improves both electric and thermal conductivities by reducing bulk and contact resistances. Slight compressions may also reduce mass transport resistance due to the shortening of the diffusion path to be covered by the reactants and products in their way to/from the catalyst layers. However, excessive compression loads may impede reactant and product trans port due to the loss of pore volume, which is typically accompanied by a reduction of the effective species diffusiv ities. On top of that, excessive assembly pressures are known to damage typical paper type GDLs, induce local delamination of the GDL under the channel, and result in non uniform compressive loads which may degrade the membrane. Pore size reduction may also affect multiphase capillary transport phenomena in the GDL (liquid water removal in PEMFCs [31] and gaseous $\mathrm{CO}_{2}$ transport in DMFCs). And last, but not least, partial GDL intrusion into the channel produces a reac tant flow rate reduction, or, alternatively, an increase of the parasitic power required to maintain the flow, which affects the overall efficiency of the stack. The main aim of GDL compression studies is thus to clarify the interplay between all these competing effects and to identify the optimal value of the compression assembly pressure that maximizes overall fuel cell performance [32].

Even though the fuel cell community has long recognized the influence of GDL compression on fuel cell performance [33-35], early modeling studies neglected this fact due to the lack of experimental data on the effects of compression on porosity, permeability, and interfacial contact resistances (see, e.g., [36] and references therein). In the last few years, however, the situation has changed, and an ever increasing attention has been paid to the characterization of this phenomenon.

Focussing the attention on previous modeling efforts, Chu et al. [37] studied the effect of through plane porosity variations by comparing four different predefined GDL porosity profiles: a constant model, a linear model, and two exponential models with different convexities. Roshandel et al. [38,39] considered the effect of compression on the porosity distribution by allowing the porosity $\phi$ to vary as a predefined periodic function of the in plane coordinate $x$ of the form $\phi_{0} \sum A_{n} \sin ^{2 n} x$ where $\phi_{0}$ was the initial porosity, and the expansion coefficients $A_{n}$ were selected as a function of compression pressure based on experimental results reported in the literature. As a result of their analysis they concluded that a non uniform catalyst loading distribution would improve the performance of the catalyst layer by diminishing current density variations. Sui et al. [40] carried out 2D numerical simulations of the coupled electrical conduction and mass diffusion in the cathodic GDL of a PEMFC and reported significant compression effects by considering a constant increased electrical conductivity and reduced binary diffusivity of species under the land area. In a further step, Su et al. [41] obtained experimental data of porosity and permeability of uncompressed and compressed GDLs using a porometer, and implemented their results in a 3D numerical model. They tested three different configurations of transport properties, namely uniform uncompressed and compressed GDL properties, and inhomogeneous GDL proper ties modeled by considering different constant values of permeability and porosity under the rib and under the channel. In a comprehensive study, Nitta et al. [28] reported experi mental data of the GDL intrusion into the channel, gas permeability, in plane and through plane bulk conductivities, and contact resistances at the interfaces as a function of the compressed GDL thickness. Subsequently, a numerical model was developed to include the experimentally measured parameters as well as the effects of variable thickness and porosity [29]. Specifically, they took photomicrographs of the GDL after the assembly process and fitted the deformed contour to a polynomial function. The resulting fit was used to calculate the porosity distribution based on the hypothesis that the reduction of GDL thickness was exclusively due to loss of pore volume. The main limitation of this methodology was that the resulting porosity field did not account for porosity varia tions in the through plane direction.

Currently, the most widespread method of analysis is based on the experimental characterization of the mechanical prop erties of the GDL followed by the numerical simulation of the inhomogeneous compression phenomenon. This approach provides the porosity and contact resistance distributions after the cell assembly process, as well as the partial intrusion into the channel. As an illustrative example, Zhou et al. [42-44] used a finite element method (FEM) model including the GDL and the bipolar plate to determine the volumetric strain at each point of the deformed porous layer as well as the contact pressure profile at the rib-GDL interface. Due to the widespread avail ability of reliable FEM software packages, the correct charac terization of the mechanical properties of the GDL remains as the Achilles' heel of this otherwise powerful approach. Indeed, the mechanical characterization of the GDL exhibits large differences between authors. Thus, while the vast majority of 
studies use linear $[18,19,45-52]$ or nonlinear isotropic models [20], or even piecewise linear isotropic models (where the Young's modulus is a function of the assembly pressure range) [42], only a few consider the more realistic assumption of nonlinear orthotropic properties [53-56].

The orthotropic character of the GDL is closely related to the microstructure of the porous material [57]. Typically, the in plane arrangement of the carbon fibers results in a highly rigid quasi planar structure which is however easily deform able in the through plane direction due to the high porosity of the material. In consequence, linear isotropic models are generally not capable of reproducing the GDL inhomogeneous compression process with accuracy. Indeed, the numerical simulations to be presented below show significant differ ences in the interfacial contact pressure distributions calcu lated with the isotropic and orthotropic models. In order to avoid this kind of uncertainties in multiphysics models, the nonlinear orthotropic behavior of the GDL must therefore be characterized as closely as possible.

The present study describes a new finite element model which fully incorporates the nonlinear orthotropic mechan ical properties of the GDL, thereby improving the prediction of the inhomogeneous compression effects in this key element of the cell. A special modeling effort has been made to char acterize the nonlinear through plane mechanical behavior of the porous material in the full deformation range by combining previous experimental results (including those reported by Kleemann et al. [54] and used by Serincan et al. $[55,56]$, and those reported by Mathias et al. [34] and used by Zhou et al. [20]) that covered smaller deformation ranges. The proposed model has been validated against experimental measurements and numerical simulations reported in the open literature, showing good agreement in both cases.

The paper is organized as follows. The numerical model is presented in Section 2. The validation against experimental and numerical results is presented in Section 3. Numerical results are presented in Section 4, including a comparison between linear isotropic and nonlinear orthotropic models, as well as a parametric study of the different geometrical and mechanical parameters characterizing the GDL. Finally, the conclusions are given in Section 5 .

\section{Numerical model}

In this section, we present the numerical model along with the underlying assumptions, including the assumed geometry, the boundary and contact conditions, and the mechanical characterization of the GDL. The model is implemented and solved using the commercial finite element code ABAQUS ${ }^{\circledR} /$ Standard [58], which is well suited for large deformation problems and allows the implementation of user defined materials. In all cases the simulations were carried out under quasi static conditions, as corresponds to the smooth assembly process of fuel cell stacks.

\subsection{Geometry and boundary conditions}

We shall assume that the region under study is located far enough from the cell boundaries so that edge effects can be ignored, and that the channels are sufficiently long to consider plane strain conditions in the streamwise direction $z\left(\begin{array}{ll}\varepsilon_{z} & 0\end{array}\right)$. Accordingly, the assembly process can be described using the 2D unit cell model depicted in Fig. 1, which includes the whole thickness of the GDL and a sufficiently large portion of the rib. As seen in the figure, the assumed GDL geometry is completely defined by three geometrical parameters, namely the rib half width, $w_{\text {rib }}$, the GDL thickness, $t_{\mathrm{GDL}}$, and the distance between the rib and channel symmetry planes, $w_{\mathrm{GDL}}$. Notice that due to the repetitive channel/rib pattern the model has been restricted to the domain limited by the mid planes of one channel and the neighboring rib, assuming symmetry conditions at both boundaries $\left(\begin{array}{ll}u_{x} & 0\end{array}\right)$. These hypotheses were validated against the results obtained with an extended 3D model which included 10 ribs compressing the porous layer. Only the region neighboring the border in direction $\mathrm{z}$ suffered noticeable strains along the $z$ axis, while the displacements in direction $x$ at the mid planes of the channels and ribs were approximately zero over the whole model width.

During the model setup process we also carried out 2D simulations in an extended domain to analyze the coupled response of the GDL and the membrane, which was charac terized using the mechanical elasto plastic properties of Nafion ${ }^{\circledR} 112$ in standard assembly conditions (i.e. $25^{\circ} \mathrm{C}$ and $30 \%$ relative humidity) reported by Kusoglu et al. [19]. For simplicity, the hydration level of the membrane was assumed to be constant throughout the compression process, although in real applications this may not be necessarily true. Due to the higher stiffness of the membrane $\left(E_{\text {mem }} \sim 200 \mathrm{MPa}\right.$ in standard assembly conditions) compared to that of the GDL (about 10-20 MPa in the through plane direction) the simu lations showed displacements at the membrane-GDL inter face of the order of a few $\mu \mathrm{m}$, which were negligible when compared with the typical vertical displacements suffered by the GDL, of the order of several tens of $\mu \mathrm{m}$. Accordingly, in the rest of the paper we shall restrict the displacement in the $y$ direction $\left(\begin{array}{ll}u_{y} & 0\end{array}\right)$ at the lower boundary of the porous layer.

The assembly process will be modeled as an imposed vertical displacement at the top of the rib, since due to the high Young's modulus of the gaskets (in the order of GPa $[46,47]$ ) the

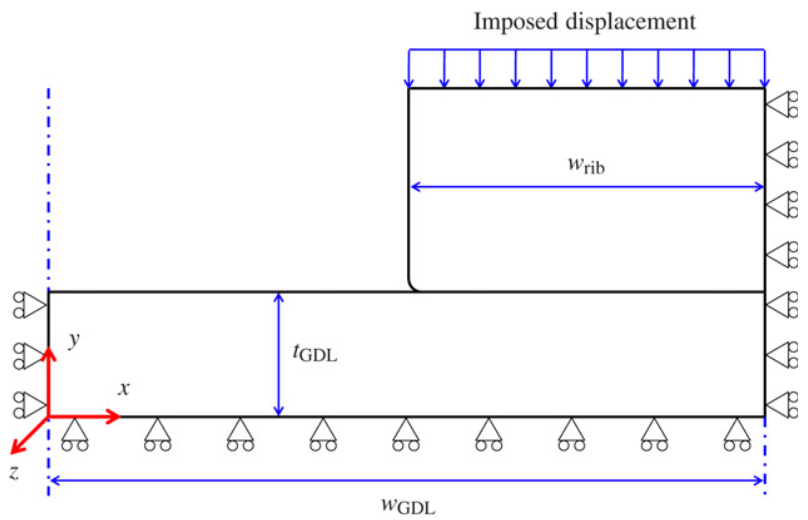

Fig. 1 - Schematic of the modeling domain showing the coordinate system, the boundary conditions, and the notation used for the rib half-width, $w_{\text {rib }}$, the GDL thickness, $t_{\mathrm{GDL}}$, and the distance between the rib and channel symmetry planes, $w_{\mathrm{GDL}}$. 
MEA is typically compressed down to the gasket thickness regardless of the compressive load. The alternative of modeling the assembly process by an imposed compressive load consti tutes a less rigorous approach, since the contact pressure acting on the GDL is typically different from the clamping pressure acting on the stack, and may even experience significant spatial variations across the active area of the cell [30].

\subsection{Rib-GDL contact condition}

A surface to surface contact condition was introduced at the rib-GDL interface. Since sliding between the rib and the GDL was expected to be negligible, a small sliding contact formu lation with a 0.2 friction coefficient [59] was implemented to describe the tangential behavior (penalty friction formulation).

In addition, a small fillet radius $(20 \mu \mathrm{m})$ was introduced at the lower corner of the rib to smooth out the singularity introduced by the presence of a right angle, thus avoiding the numerical problems that would arise otherwise. As shown in Section 4.4, simulations varying the fillet radius show that the influence to this parameter is purely local (in terms of the rib-GDL contact pressure distribution), and does not affect the overall nature of the solution.

\subsection{Materials}

The mechanical properties of the (graphite) bipolar plate were assumed to be isotropic, with a Young's modulus E $10 \mathrm{GPa}$ and a Poisson ratio $\nu$ 0.25, while for the porous layer we considered the nonlinear orthotropic properties of carbon paper. In particular, the GDL mechanical characterization assumed in our study corresponds to Toray ${ }^{\circledR}$ carbon paper TGP H series, whose properties are widely reported in the open literature. Moreover, the following hypothesis were considered for the definition of the GDL mechanical behavior:

1. Large strain theory is required to properly reproduce the compression of the porous layer, since as previously dis cussed GDL strains under the rib area reach typical values between 10 and $40 \%$ during the cell assembly process.

2. The mechanical behavior of the heterogeneous carbon paper is described through a homogenized constitutive model, commonly used for fibrous materials.

3. As in other non woven felts, GDLs based on carbon paper typically show slightly different orientations of the fibers in the material plane, namely in the machine and cross machine directions [54]. However, in our simulations the mechanical behavior in the $x$ and $z$ directions is considered equivalent. Due to the through plane load state and the higher in plane stiffness, small strains occur in these directions (except for local effects close to the lower corner of the rib). Accordingly, linear elastic behavior is assumed in the $x$ and $z$ directions, neglecting increase of stiffness due to fiber alignment and irreversible deformation due to disentanglement.

4. The GDL material is assumed to have the same properties in tension and compression. Although the properties in the through plane direction considered in this paper corre spond to compression tests, a thin GDL region situated below the channel undergoes tensile strains, a situation which must be taken into account.

5. The response in the $y$ direction is considered elastic. Since we are only interested in the cell assembly process, the hysteretic behavior of the GDL under cyclic compression [8,16,34,60-62] has been ignored in our model. Note that the analysis of loading-unloading cycles (e.g. due to membrane swelling) would require the development of a predictive model that includes path dependence effects. As a first approximation, one could resort to theoretical pseudo elastic models [63] developed for other materials exhibiting hysteretic behavior, such as polymeric foams [64], and try to apply the same ideas to reproduce the distinctive features shown by carbon papers submitted to cyclic loads [65].

6. The shear modulus $G_{x y}$ is assumed to be constant up to failure $[53,54]$.

7. Poisson's ratios $\nu_{y x}$ and $\nu_{y z}$ (defined as the ratios between the strains in the $x$ and $z$ directions and the strain in the $y$ direction, respectively) are assumed to be equal to 0 , since due to the porous microstructure of the material and the high stiffness of the carbon fibers the volume reduction during compression can be attributed to the reduction of pore volume only [54].

The last hypothesis deserves further attention. Notice that if $\nu_{y x}$ is small but not exactly zero, the relationship between reciprocal Poisson's ratios $\nu_{x y} \quad\left(E_{x} / E_{y}\right) \nu_{y x}$ establishes that $\nu_{x y}$ may take values of order unity for $E_{x} / E_{y} \sim \nu_{y x}^{-1} \gg 1$, as is seen to occur in other orthotropic materials with high anisotropy between the in plane and through plane directions $[66,67]$. Thus, in order to investigate the effect of non zero values of Poisson's ratio $\nu_{y x}$ we carried out plane strain simulations for values of $\nu_{y x}$ ranging from zero up to the material stability limit, which for $\nu_{y x} \quad \nu_{y z}$ and $\nu_{x z} \quad \nu_{z x} \quad 0.25[55,56]$ restricts the possible values of $\nu_{y x}$ slightly below $10^{2}$. It is interesting to note that the simulations showed no significant influence of $\nu_{y x}$ on the numerical results, so that this parameter was thereafter taken equal to 0 .

Under the assumptions stated above, the constitutive equation for the GDL material corresponding to plane strain conditions adopts the following form using Voigt notation:

$\left[\begin{array}{l}\mathrm{d} \sigma_{x} \\ \mathrm{~d} \sigma_{y} \\ \mathrm{~d} \tau_{x y}\end{array}\right] \quad\left[\begin{array}{cccc}E_{x} /(1 & \left.\nu_{x z} \nu_{z x}\right) & 0 & 0 \\ 0 & E_{y}\left(\varepsilon_{y}\right) & 0 \\ 0 & 0 & G_{x y}\end{array}\right]\left[\begin{array}{c}\mathrm{d} \varepsilon_{x} \\ \mathrm{~d} \varepsilon_{y} \\ \mathrm{~d} \gamma_{x y}\end{array}\right]$

where $\sigma_{i}$ and $\varepsilon_{i}$ are respectively the normal true stress and the longitudinal true strain in direction $i$, and $\tau_{x y}$ and $\gamma_{x y}$ are the shear stress and strain associated to directions $x$ and $y$.

According to the results presented by Kleemann et al. [54], obtained for Toray $^{\circledR}$ carbon paper TGP H 060, the mechanical behavior of the GDL in the material $(x z)$ plane is very different to that exhibited in the through plane $(y)$ direction. Since fibers are arranged in a bidimensional structure, the behavior of the material in its plane is much stiffer than in the transverse direction, where the porosity dominates the structural response. Thus, while the elastic moduli in the material plane $\left(E_{x}\right.$ and $\left.E_{z}\right)$ are usually in the order of $\mathrm{GPa}$, in the through plane direction $\left(E_{y}\right)$ it fails to overcome tens of $\mathrm{MPa}$, similarly to the shear modulus $\left(G_{x y}\right)$, which is also in the order of several MPa. 
It is worth noting that while $E_{x}$ appears in Eq. (1) divided by a factor $1 \nu_{x z} \nu_{z x}$ to account for the effect of the non zero Poisson's ratios $\nu_{x z} \nu_{z x} \quad 0.25$, the values of the in plane Young's modulus reported throughout the paper correspond, in fact, to the first term of the diagonal in the constitutive matrix, $E_{x} /\left(1 \quad \nu_{x z} \nu_{z x}\right)$. Thus, to recover the actual value of the in plane Young's modulus one should multiply the reported value of $E_{x}$ by $1 \quad 0.25^{2} \quad 0.9375$. Due to the small influence of this parameter in the results (see, e.g., the sensitivity analysis presented in Section 4.4), the resulting differences, amounting to a few percent, have a completely negligible effect.

To characterize the nonlinear behavior of the GDL in the through plane direction, of special relevance to our study, we have compiled multiple experimental data sets from the open literature in the form of stress/strain and stress/displacement curves corresponding to TGP H $060[34,54]$ and TGP H 090 $[8,68]$ carbon papers. Fig. 2 shows the different $E_{y}\left(\varepsilon_{y}\right)$ curves obtained by numerical derivation of the true stress strain data obtained from the literature. Note that while the stress data were assumed to be reported as true stress values, since transverse deformations are negligible given the approxi mately zero Poisson's ratio $\nu_{y x}$, the strain data were assumed to be reported as engineering values (although not explicitly reported by the authors), so that they had to be converted to true strains before proceeding with the numerical derivation. As seen in the figure, the resulting $E_{y}\left(\varepsilon_{y}\right)$ curves exhibit three different regions of GDL response to compressive loads: an initial region for relatively small strains, where the material gradually hardens, an intermediate region with constant Young's modulus, and a final large strain region where the material hardens again. The nature of these three regions can

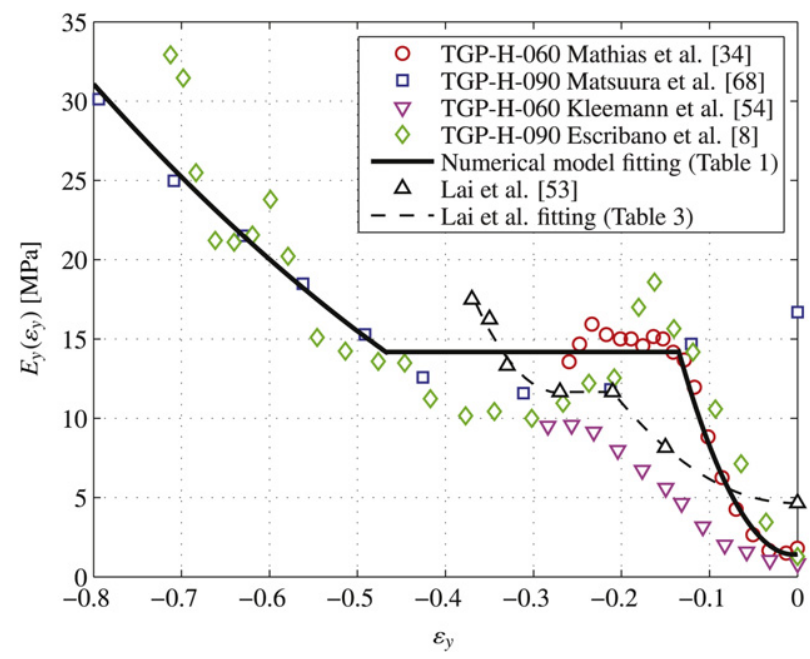

Fig. 2 - Nonlinear Toray ${ }^{\circledR}$ carbon paper TGP-H-060/090 mechanical behavior in the through-plane direction $E_{y}\left(\varepsilon_{y}\right)$ calculated by numerical derivation of the true stress/strain curve obtained from the data reported by Mathias et al. [34] and Matsuura et al. [68] (20 wt. \% PTFE), and the stress/ displacement data reported by Kleemann et al. [54] and Escribano et al. [8] (10 wt. \% PTFE). The figure also shows the $E_{y}\left(\varepsilon_{y}\right)$ curve constructed with the stress/strain data reported by Lai et al. [53] and the corresponding polynomial fit. be understood based on arguments found in the literature. The first region has been attributed both to the flattening of the GDL surface asperities [30] and to the increased number of contacts among fibers caused by the initial closure of pores [7]; the constant region may be traced back to the intrinsic behavior of the microstructure of the porous layer; and the large strain hardening region is most likely due to the final collapse of the GDL and the resulting increase in contacts between fibers at high pore volume reductions [69]. Note that even though the PTFE content in the different GDLs reviewed in Fig. 2 is different, the nonlinear mechanical behavior is qualitatively similar in all cases.

To implement the nonlinear mechanical behavior of the GDL in our numerical model we used the piecewise poly nomial fit reported in Table 1, obtained from the experimental data by Mathias et al. [34] and Matsuura et al. [68]. Shown as a thick black line in Fig. 2, the piecewise polynomial fit exhibits the three regions that characterize the mechanical behavior of the GDL in the through plane direction, and provides a good quantitative description for the GDL response under compression.

Table 2 summarizes the mechanical properties for Toray ${ }^{\circledR}$ carbon paper TGP $\mathrm{H}$ series used as reference case in our study. Except for the nonlinear behavior $E_{y}\left(\varepsilon_{y}\right)$ in the through plane direction, the remaining properties correspond exclusively to the Toray ${ }^{\circledR}$ TGP H 060 data reported by Kleemann et al. [54]. Other types of carbon papers and carbon cloths could simi larly be modeled as nonlinear orthotropic materials using the corresponding mechanical properties obtained experimen tally [34,54].

The constitutive equation of the GDL was implemented in the form given in Eq. (1) through the user subroutine UMAT, provided by ABAQUS ${ }^{\circledR} /$ Standard for the customization of mechanical constitutive models. It is worth noting that due to the nonlinear mechanical response of the GDL in the through plane direction, a sufficiently small step increment had to be chosen during the simulations in order to reproduce the variation in the mechanical properties at each stage of the deformation process.

\subsection{Element discretization and grid-independence study}

Four node bilinear plane strain quadrilateral elements (CPE4) were used in the simulations. To check the accuracy of the numerical solution, a grid refinement study was performed for the reference geometry of $190 \mu \mathrm{m}$ GDL thickness and $1000 \mu \mathrm{m}$

Table 1 - Piecewise polynomial fitting of the throughplane nonlinear behavior for Toray ${ }^{\circledR}$ carbon paper TGP-H060/090.

\begin{tabular}{lll} 
Region & Polynomial fitting [MPa] & Domain \\
\hline $\begin{array}{c}\text { Small strain } \\
\text { hardening }\end{array}$ & $745.00 \varepsilon^{2}+5.87 \varepsilon+1.42$ & $0.135<\varepsilon \leq 0$ \\
$\begin{array}{c}\text { Constant } \\
\text { modulus }\end{array}$ & 14.175 & $0.47<\varepsilon \leq 0.135$ \\
$\begin{array}{c}\text { Large strain } \\
\text { hardening }\end{array}$ & $33.23 \varepsilon^{2} \quad 8.70 \varepsilon+2.84$ & $\varepsilon \leq 0.47$ \\
\begin{tabular}{c} 
Tensile \\
\hline
\end{tabular} & Symmetrical (even function) & $\varepsilon>0$ \\
\hline
\end{tabular}


Table 2 - Toray $^{\circledR}$ carbon paper TGP-H series mechanical properties.

\begin{tabular}{llll} 
Parameter & \multicolumn{1}{c}{ Value } & \multicolumn{1}{c}{ GDL } & Reference \\
\hline$E_{y}\left(\varepsilon_{y}\right)$ & Numerical & TGP H 060/090 & {$[8,34,54,68]$} \\
& fitting (see Table 1) & & \\
$E_{x}$ & $7 \mathrm{GPa}$ & TGP H 060 & {$[54]$} \\
$E_{z}$ & $7 \mathrm{GPa}$ & TGP H 060 & {$[54]$} \\
$G_{x y}$ & $18.5 \mathrm{MPa}$ & TGP H 060 & {$[54]$} \\
\hline
\end{tabular}

channel and rib widths. At each refinement level, a refined grid was obtained by halving the element size of the baseline grid. Grid independence was achieved with a $2 \mu \mathrm{m}$ element size, which showed an average relative error of order $10^{4}$ in the numerical evaluation of the porosity field as compared with the $1 \mu \mathrm{m}$ refined grid, so this level of accuracy was considered appropriate.

\section{Model validation}

Before proceeding further, the model was validated against the numerical simulations and experimental results reported by Lai et al. [53] and Kandlikar et al. [45], respectively. The representative variable used to compare the results was the partial intrusion into the channel, defined as the difference between the compressed GDL thickness at the channel symmetry plane and the compressed GDL thickness under the rib. During the validation campaign, the simulations were carried out by imposing the assembly pressure instead of the displacement onto the rib, since the available data was also reported in this way.

Lai et al. [53] studied numerically the effect of intrusion on the performance of PEMFCs. To this end, they characterized the mechanical behavior of various GDLs (brand names not reported) in compressive, flexural, and shear tests and used their results in a numerical model to calculate the channel intrusion. Subsequently, they developed a simplified reactant flow redistribution model of parallel channels to estimate the effect of channel intrusion on the reactant flow redistribution. In their simulations of the assembly process they implemented the bipolar plate as a rigid surface. Accordingly, we modeled the bipolar plate in this way so as to mimic their results as closely as possible. Moreover, they modeled the nonlinear orthotropic behavior of the GDL by superposing two element types together (gasket and plane strain elements). The compressive stress/strain curve from the compressive test was used to model the behavior of the gasket elements, while the solid elements were assigned orthotropic elastic properties with a very small through plane Young's modulus. Fig. 2 shows the stress/strain curve constructed with the data reported by Lai et al. [53] and the polynomial fit to the Young's modulus $E_{y}\left(\varepsilon_{y}\right)$. The $E_{y}\left(\varepsilon_{y}\right)$ curve follows a similar behavior to that seen for Toray ${ }^{\circledR}$ carbon paper TGP H series, although the region of constant Young's modulus is quite small in this case. The piecewise polynomial fitting for the Young's modulus is summarize in Table 3 . It should be noted that the data set presented by Lai et al. is very small, so that a larger amount of data would be required for a more accurate fit of the nonlinear
Table 3 - Piecewise polynomial fitting of the throughplane nonlinear behavior corresponding to the data reported by Lai et al. [53].

\begin{tabular}{lll} 
Region & Polynomial fitting [MPa] & Domain \\
\hline $\begin{array}{c}\text { Small strain } \\
\text { hardening }\end{array}$ & $166.67 \varepsilon^{2}+1.67 \varepsilon+4.67$ & $0.21<\varepsilon \leq 0$ \\
$\begin{array}{c}\text { Constant } \\
\text { modulus }\end{array}$ & 11.67 & $0.27<\varepsilon \leq 0.21$ \\
$\begin{array}{c}\text { Large strain } \\
\text { hardening }\end{array}$ & $630.18 \varepsilon^{2}+341.68 \varepsilon+57.93$ & $\varepsilon \leq 0.27$ \\
Tensile & Symmetrical (even function) & $\varepsilon>0$ \\
\hline
\end{tabular}

behavior of the material. The elastic moduli from the flexural test and the apparent shear moduli from the shear test were used as in plane Young's moduli $E_{x}$ and $E_{z}$ and shear modulus $G_{x y}$ respectively. Finally, since the GDL has a very high porosity, a very small Poisson's ratio was considered (which was assumed zero in our simulations). The values of the geometric dimensions and mechanical properties used to validate the model against the numerical results presented by Lai et al. [53] are as follows: $w_{\mathrm{GDL}} \quad 1500 \mu \mathrm{m}, w_{\text {rib }} \quad 1000 \mu \mathrm{m}, t_{\mathrm{GDL}} \quad 260 \mu \mathrm{m}$, $E_{x} \quad 0.3 \mathrm{GPa}, E_{z} \quad 0.9 \mathrm{GPa}, G_{x y} \quad 9.2 \mathrm{MPa}$.

The comparative between the results obtained with our model and those reported by Lai et al. [53] is shown on the left plot of Fig. 3. It can be seen that both curves exhibit an analo gous behavior, providing similar intrusion values in all the assembly pressure range. The small differences observed may be attributed to the different methodology used to describe the nonlinear behavior in the through plane direction.

The work carried out by Kandlikar et al. [45] focused on the experimental measurement of the intrusion in parallel channels and its effect on the flow distribution. The intrusion was determined as a function of the assembly pressure using two different methods: an optical measurement method and an analytical fluid flow model based on individual channel flow rate measurements. Based on these intrusion measure ments, they estimated the Young's modulus of the GDL by means of a finite element (ANSYS ${ }^{\circledR}$ ) linear isotropic model. The GDL used in their study was Toray ${ }^{\circledR}$ carbon paper TGP H 060, so that the mechanical properties assumed in the vali dation simulations were those presented in Table 2. Moreover, the FEM model geometry was adapted so as to reproduce the experimental conditions in [45]: $w_{\mathrm{GDL}} \quad 600 \mu \mathrm{m}, w_{\mathrm{rib}} \quad 250 \mu \mathrm{m}$, $\mathrm{t}_{\mathrm{GDL}} \quad 230 \mu \mathrm{m}$.

The right plot of Fig. 3 presents the intrusion measure ments reported by Kandlikar et al. [45] together with the numerical predictions obtained with our model. It is inter esting to note that the GDL considered in [45] included a microporous layer (MPL) which was not present in the numerical model. As can be seen, the proposed model provides intrusion values near the upper experimental limit in all the assembly pressure range. This is an expected result, since the presence of the MPL is known to increase the stiff ness of the GDL (see, e.g., [42]) and higher stiffness tends in turn to reduce GDL intrusion, so that the observed differences are qualitatively correct. Since the intrusion values were in the same order of magnitude and the behavior was appropriate, specially when taking into account the presence of the MPL, the validation of our model was considered satisfactory. 

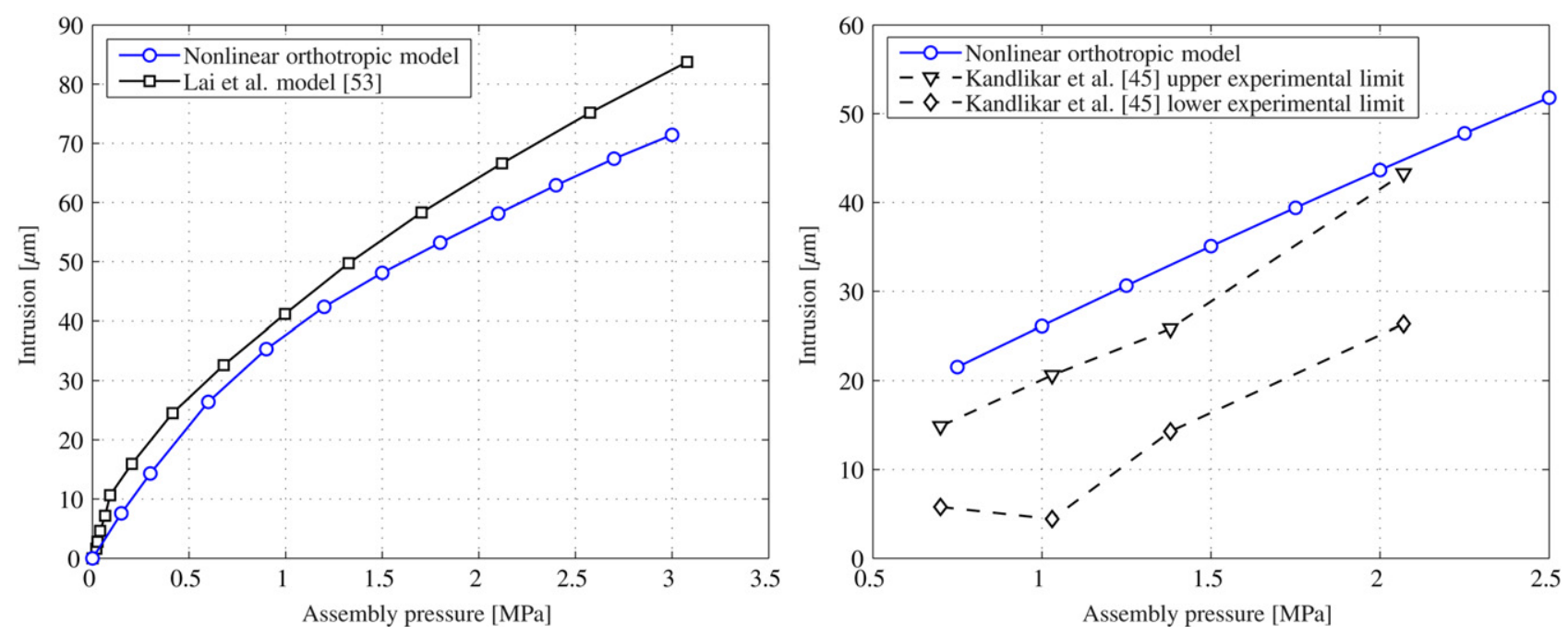

Fig. 3 - Variation of the GDL intrusion into the channel as a function of the cell assembly pressure. Left plot: numerical results obtained with the proposed nonlinear orthotropic model and the numerical model of Lai et al. (53). Right plot: numerical predictions of the nonlinear orthotropic model for $t_{\mathrm{GDL}}=230 \mu \mathrm{m}$ (solid line) and upper and lower experimental limits reported by Kandlikar et al. [45] for a GDL thickness of $230 \mu \mathrm{m}$ including a MPL of $40 \mu \mathrm{m}$ (dashed lines).

\section{Results and discussion}

The numerical results are presented in four different sections. Section 4.1 is devoted to the calculation of the porosity field and the corresponding effective diffusivities, Section 4.2 illustrates the spatial variations of the through plane Young's modulus $E_{y}$ as a function of the assembly load, Section 4.3 compares the predictions of the nonlinear ortho tropic model presented in this paper with those of linear isotropic models reported in the literature, and Section 4.4 presents a sensitivity analysis of the effect of the different geometrical and mechanical parameters in the inhomoge neous compression phenomenon.

The case under study in the first three sections consti tutes the reference case for the sensitivity analysis. Specifi cally, the reference geometry corresponds to $w_{\mathrm{GDL}} \quad 1000 \mu \mathrm{m}$ (assumed constant throughout the study) and $w_{\text {rib }} 500 \mu \mathrm{m}$, while the reference mechanical properties are those of Toray ${ }^{\circledR}$ carbon paper TGP H series presented in Table 2. Since all mechanical properties (except the nonlinear behavior $\left.E_{y}\left(\varepsilon_{y}\right)\right)$ correspond to carbon paper TGP H 060, the reference thickness and initial porosity have been set equal to the nominal values $t_{\mathrm{GDL}} \quad 190 \mu \mathrm{m}$ and $\phi_{0} \quad 0.8$ reported in the product datasheet [70]. Note that the porosity reported by the manufacturer for TGP H 060 is 0.78 , but for the qualitative purpose of our study it has been rounded to 0.8 .

In the simulations of the assembly process, the maximum imposed vertical displacement at the top of the rib was set equal to $32 \%$ of the initial GDL thickness in all cases, except for the study dedicated to the spatial distribution of the through plane Young's modulus $E_{y}$, in which the maximum displace ment was set to $42 \%$ of the initial thickness to ensure the presence of the three mechanical response regions of the curve $E_{y}\left(\varepsilon_{y}\right)$ during the loading process.
It is worth noting that the different contour plots presented below always use the same color scale for the same variable (i.e. porosity, effective diffusivity, and through plane Young's modulus) thus facilitating visual comparisons of the effect of the different parameters.

\subsection{Porosity and effective diffusivity fields}

Assuming that the volume changes experienced by the GDL are due solely to the decrease of pore volume, the calculation of the porosity field $\phi(x, y)$ after the fuel cell assembly process is given, in the context of large strain theory, by the following expression $[43,44]$

$\phi(x, y) \quad \frac{V_{p}}{V} \quad \frac{\phi_{0} 1+e^{\varepsilon_{V}(x, y)}}{e^{\varepsilon_{V}(x, y)}}$

where $\phi_{0}$ is the initial porosity, $V_{p}$ the final pore volume, $V$ the final total volume, and $\varepsilon_{\mathrm{V}}(\mathrm{x}, \mathrm{y})$ the true volumetric strain at each point of the elastic material.

Fig. 4 illustrates the changes suffered by the porosity field during the compression process as the imposed vertical displacement increases from 0 to $60 \mu \mathrm{m}$. The GDL intrusion into the channel and the compressive stress applied at the rib symmetry plane are also indicated for illustrative purposes. The inhomogeneity associated with the repetitive channel rib pattern is perfectly reflected, showing a region of large porosity reduction (about 12\% for the largest imposed displacement) under the rib, a region of unperturbed porosity under the channel, and an intermediate fan like transition region below the channel rib wall. Note in particular the accumulation of stresses under the rib corner, which results in high porosity reduction in this particular region. The simulations also show that the upper edge of the GDL expe riences slight tensile strains in the region below the channel, 

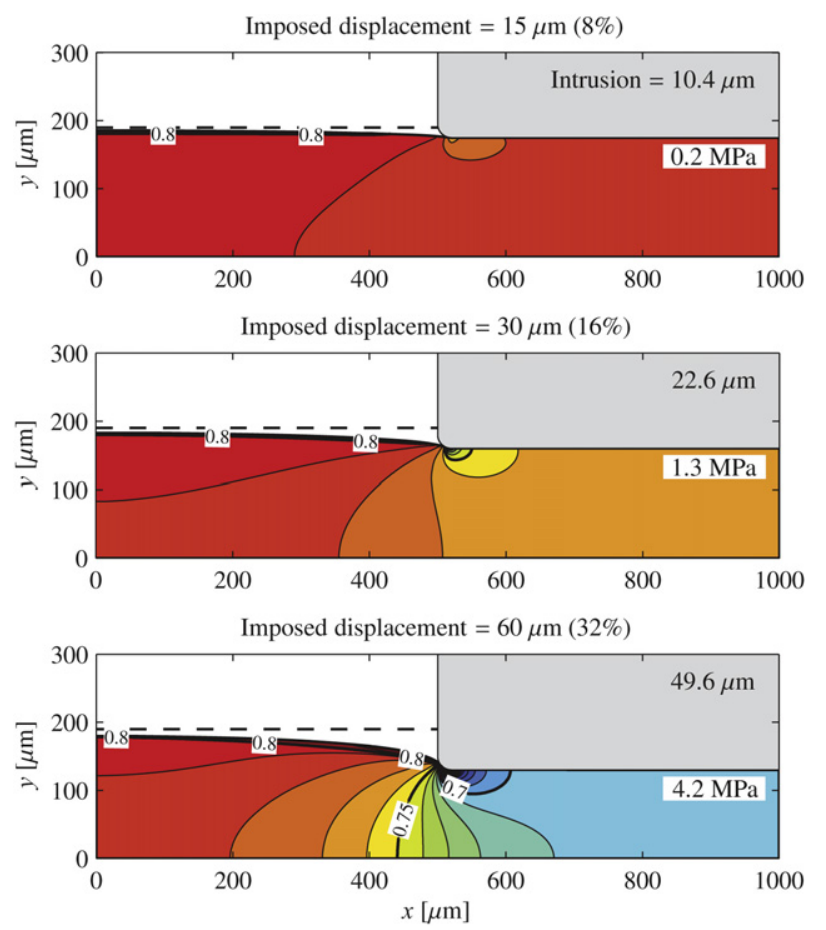

Fig. 4 - Porosity field $\phi(x, y)$, GDL intrusion into the channel, and compression stress at the rib symmetry plane obtained for different imposed rib displacements (expressed both in $\mu \mathrm{m}$ and as a percentage of the initial GDL thickness, $t_{\text {GDL }}=190 \mu \mathrm{m}$ ) and for an initial porosity $\phi_{0}=0.8$.

thereby increasing the GDL porosity above its initial value $\phi_{0} \quad$ 0.8. As a final remark, we note that the visual appearance of the porosity distribution is very similar to that calculated by Zhou et al. [42, Fig. 9] with a piecewise linear isotropic model.

To evaluate the effect of the obstruction caused by the pore walls on the diffusion fluxes of the chemical species in the GDL we shall refer to the experimental data obtained by Flückiger et al. [71,72] and Möst et al. [73] using a novel electrochemical diffusimetry method [74]. These authors have characterized the anisotropic effective diffusivities as a function of compression for different GDL types and hydrophobic treatments.

To give mathematical expression to their results, we fitted the reported effective in plane (ip) and through plane (tp) diffusivities for carbon paper TGP H 060 of various PTFE contents to the exponential functions

$\frac{D_{i, j}^{\text {eff,ip }}}{D_{i, j}} \quad \frac{\phi}{\tau_{\text {ip }}} \simeq 0.029 \exp (3.8 \phi)$

$\frac{D_{i, j}^{\text {eff,tp }}}{D_{i, j}} \quad \frac{\phi}{\tau_{\mathrm{tp}}} \simeq 0.0065 \exp (5.021 \phi)$

where $D_{i, j}$ is the binary diffusivity of species $i$ in species $j, D_{i, j}^{\text {eff }}$ the effective binary diffusivity of those species in the porous material, and $\tau$ is the tortuosity. Due to the in plane arrange ment of the fibers the pores are preferentially oriented in this direction, which results in lower tortuosity $\left(\tau_{\text {ip }}<\tau_{\text {tp }}\right)$ and thus higher effective diffusivity $\left(D^{\text {eff,ip }}{ }_{i, j}>D^{\text {eff,tp }}{ }_{i, j}\right)$. The above expressions show indeed that the anisotropy between the in plane and through plane directions is about 2 and increases as the GDL is compressed due to the realignment of fibers. It is worth noting that the isotropic spherical agglom erate model of Bruggeman [75] and the more realistic aniso tropic random fiber model of Tomadakis and Sotirchos [76], widely used in the literature, tend to oversimplify the complex geometry of real GDLs. Both models ignore, for example, the influence of structural irregularities such as binder and PTFE $[71-73,77,78]$, resulting in an overestimation of the effective diffusivity by a factor of about 2 [71-73].

Fig. 5 illustrates the effective in plane and through plane diffusivities obtained from the empirical correlations (3) and (4) corresponding to an imposed vertical displacement of $60 \mu \mathrm{m}$. The effective diffusivity predicted by Bruggeman correction, $D_{i, j}^{\text {eff }} / D_{i, j} \phi^{1.5}$, is also shown for comparative purposes. As can be seen, the numerical values are quite different depending on the considered model. Thus, while the theory of Bruggeman predicts a moderate isotropic diffusivity reduction below the rib (about 0.6), the empirical correlations (3) and (4) yield more severe reductions (about 0.43 and 0.23 for the in plane and through plane values, respectively). Note also that due to the exponential character of the correlations, relatively small porosity inhomogeneities (about 12\% of the initial porosity) lead to strong spatial variations of the effec tive diffusivities (between $30 \%$ and $40 \%$ of the unperturbed in plane and through plane values, respectively). Future fuel cell modeling efforts should therefore account for the effects of variable porosity and use accurate descriptions of the
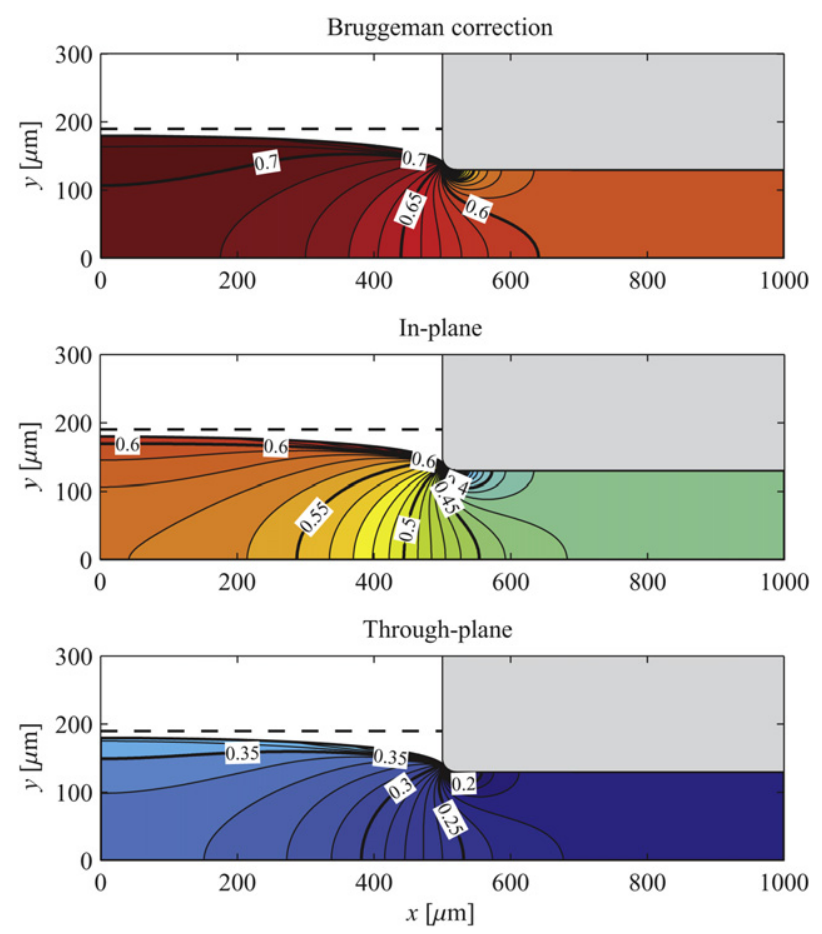

Fig. 5 - Effective diffusivity estimated from Bruggemman correction (upper plot) and effective in-plane and troughplane diffusivities predicted by the empirical correlations given in Eqs. (3) and (4) (lower plots) for an imposed rib displacement of $60 \mu \mathrm{m}$ (32\% of the initial GDL thickness, $\left.t_{\mathrm{GDL}}=190 \mu \mathrm{m}\right)$. 
anisotropic effective diffusivities in order to increase their predictive capabilities.

\section{2. $\mathrm{E}_{\mathrm{y}}$ field}

Fig. 6 shows the variation of the through plane Young's modulus $E_{y}\left(\varepsilon_{y}(x, y)\right)$ with the imposed vertical displacement, illustrating the changes in the mechanical response of the GDL during the assembly process. At first, for small loads, the Young's modulus $E_{y}$ is very small throughout the porous layer, since the whole GDL is at the initial hardening state. However, when the applied load increases, $E_{y}$ becomes highly inhomo geneous. Thus, while the area under the rib continues to harden until it reaches the constant region of the $E_{y}\left(\varepsilon_{y}\right)$ curve, the channel region remains at its initial unperturbed state. At higher compressions, three distinguished regions eventually appear. The area under the rib, with a Young's modulus that reaches the region of large strain hardening of the $E_{y}\left(\varepsilon_{y}\right)$ curve, a transition region below the channel rib wall, where the Young's modulus is constant, and the region under the channel, with $E_{y}$ still at the initial hardening state.

\subsection{Isotropic models vs. nonlinear orthotropic models}

To study the effect of the nonlinear orthotropic behavior of the GDL in the inhomogeneous compression phenomenon, the nonlinear orthotropic model was compared with two linear isotropic models found in the literature. The first one
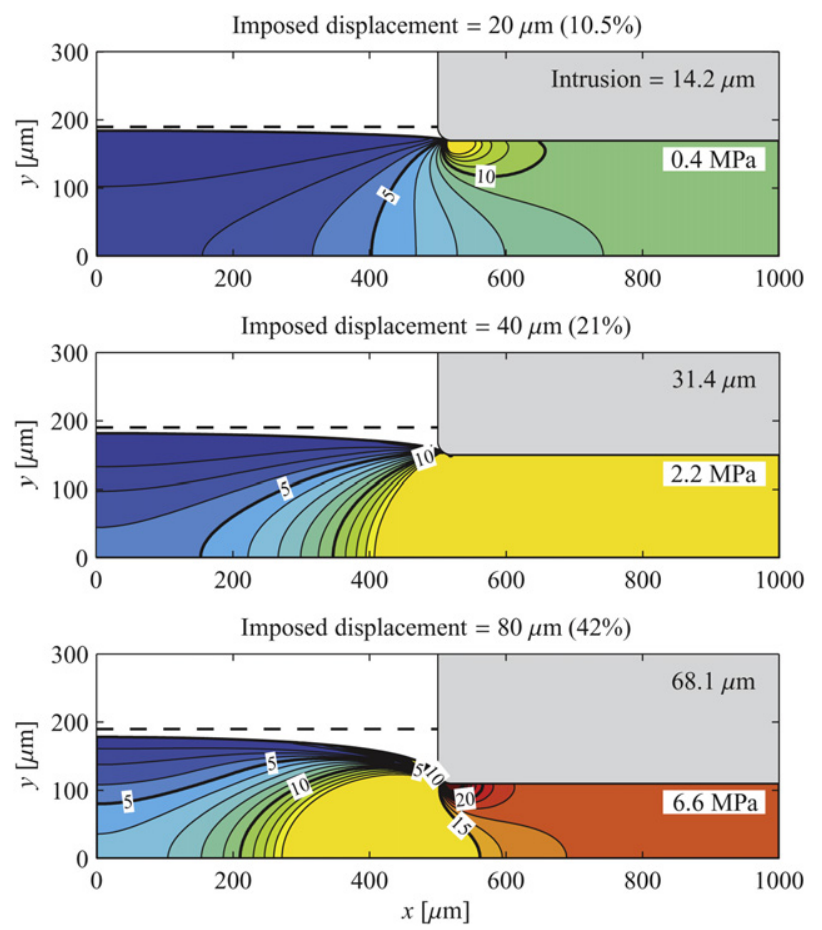

Fig. 6 - Distribution of through-plane Young's modulus $E_{y}$ [MPa] obtained for different imposed rib displacements (expressed both in $\mu \mathrm{m}$ and as a percentage of the initial GDL thickness, $t_{\mathrm{GDL}}=190 \mu \mathrm{m}$ ) and for an initial porosity $\phi_{0}=0.8$. Note that the imposed displacements are slightly larger than those considered in Fig. 4.
(E $6 \mathrm{MPa}, \nu$ 0.1) was used in Refs. $[48,49,50,52]$, while the second one ( $E \quad 18 \mathrm{MPa}, \nu$ 0) was initially proposed by Kand likar et al. [45].

Fig. 7 shows comparative results in terms of the porosity field predicted by the three models under study for the same loading conditions. As can be seen, the porosity distributions predicted by the linear isotropic models are virtually identical. Moreover, the nonlinear orthotropic model yields similar results, with small differences on the order of $1 \%$ in the region below the channel, where the linear models tend to over estimate the porosity. It can therefore be concluded that the porosity distribution is rather insensitive to the mechanical characterization of the GDL. By contrast, noticeable differences are observed when comparing the deformed GDL shapes: while linear isotropic models remain virtually unaffected below the channel, the nonlinear orthotropic model predicts significant downward displacements in this region. As a consequence, the nonlinear orthotropic model is seen to predict smaller intru sions than linear isotropic models, for which the intrusion coincides indeed with the imposed rib displacement.

Further differences can be observed in the contact pressure distributions at the rib-GDL and membrane-GDL interfaces, shown in Fig. 8. For the load condition under study (imposed rib displacement), the maximum contact pressure is located under the rib and increases with the local value of the Young's modulus in the through plane direction. Accordingly, the
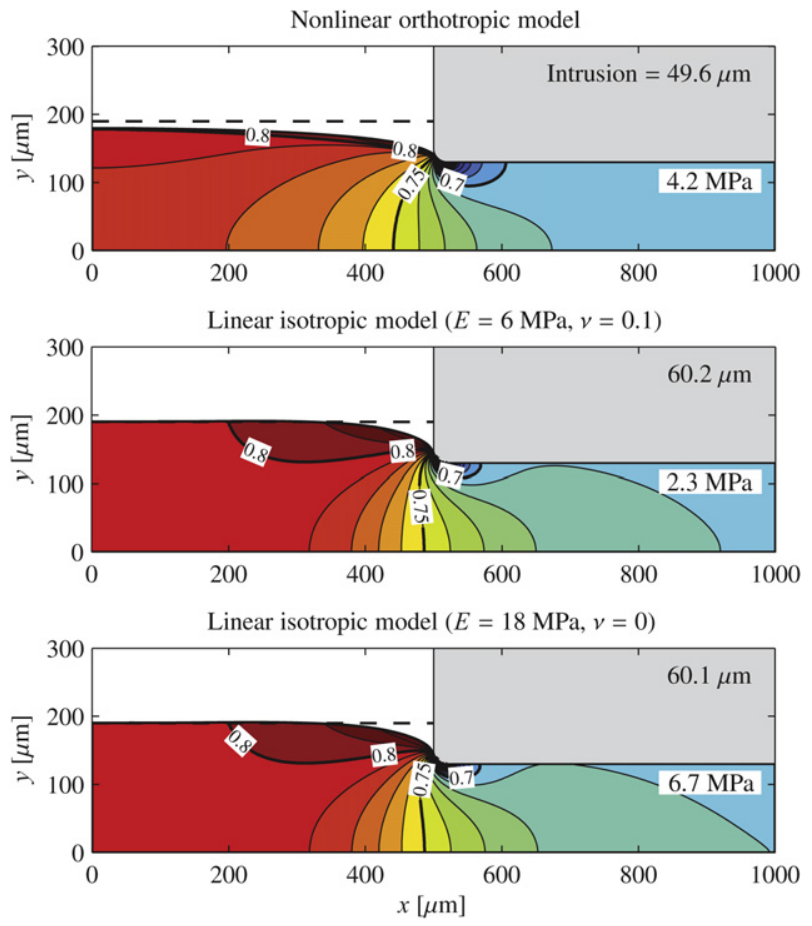

Fig. 7 - Porosity field $\phi(x, y)$, GDL intrusion into the channel, and compression stress at the rib symmetry plane obtained with the nonlinear orthotropic model and two linear isotropic models presented in the literature for an imposed rib displacement of $60 \mu \mathrm{m}$ (32\% of the initial GDL thickness, $\left.t_{\mathrm{GDL}}=190 \mu \mathrm{m}\right)$. The first isotropic model $(E$ $=6 \mathrm{MPa}, \nu=0.1$ ) corresponds to that used in Refs.

[48-50,52], while the second one $(E=18 \mathrm{MPa}, \nu=0)$ was initially proposed by Kandlikar et al. [45]. 


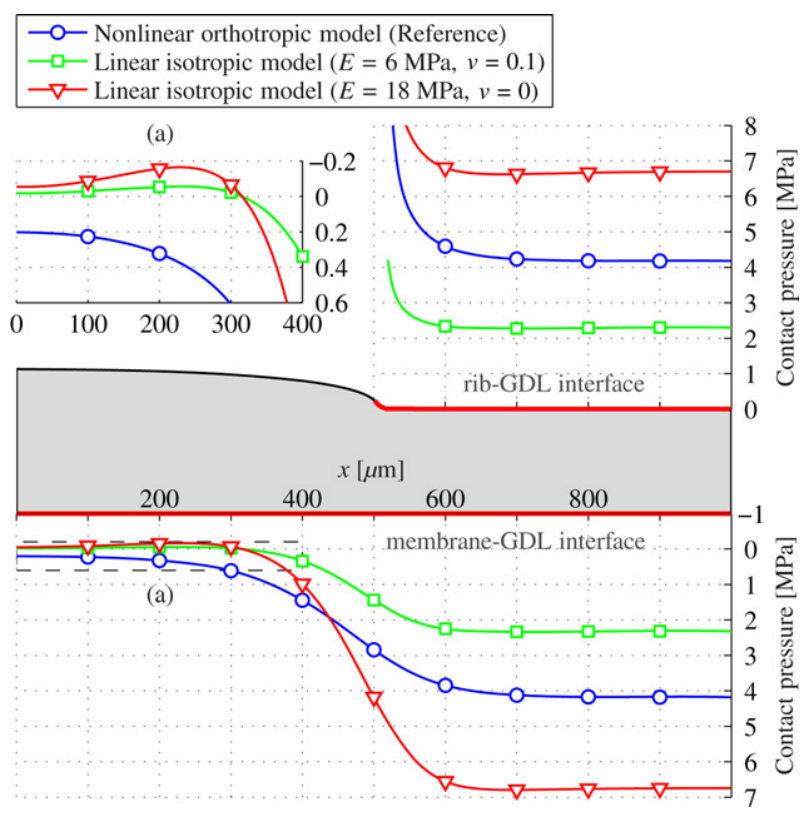

Fig. 8 - Contact pressure distribution at the rib-GDL (top) and membrane-GDL (bottom) interfaces corresponding to the three models reported in Fig. 7. The inset (a) shows a close-up view of the contact pressure at the membrane-GDL interface in the region below the channel. Note the inverse vertical scale of the lower plot.

higher contact pressures occur in the isotropic model with $18 \mathrm{MPa}$ Young's modulus and the lower in the isotropic model with $6 \mathrm{MPa}$ Young's modulus. The nonlinear orthotropic model, where $E_{y}\left(\varepsilon_{y}\right)$ reaches the constant modulus region ( $E_{y} \quad 14.175 \mathrm{MPa}$ ) for the imposed rib displacement of $32 \%$ of the initial GDL thickness, predicts intermediate contact pres sures. Note also that the transmission of the compressive loads from the rib to the membrane is much more gradual in the nonlinear orthotropic model, where the contact pressure profile is smoother and penetrates further into the channel. In fact, linear isotropic models are seen to predict small negative GDL membrane contact pressures (i.e. tensile stresses) in the region below the channel, while the nonlinear orthotropic model predicts compressive loads everywhere.

This difference may be explained based on the extremely high in plane Young's modulus $E_{x} \quad E_{z}$ of the GDL compared to its through plane value $E_{y}$. Due to the high in plane stiffness of the material, the upper edge of the GDL acts as a shell under tensile stress which pulls its left side downwards without experiencing resistance from the small through plane stiff ness of the material. This goes hand in hand with the compressive stresses arising below the channel and results in intrusions that are smaller than the imposed rib displacement. The situation is drastically different for the isotropic material, where the through plane stiffness opposes an important resistance to the downward displacement in the region close to the rib corner. This somehow pushes the material towards the channel, inducing an in plane compressive state that leads to a slight upward displacement on the left side of the GDL. In this case the boundary condition considered in the model for the lower edge (Fig. 1) generates tensile stresses on the left side to avoid this displacement. Anyhow, the magnitude of these tensile stresses is negligible as compared to the level of the contact pressures under the rib.

In summary, while the porosity field is rather insensitive to the constitutive model, the nonlinear orthotropic behavior of the porous layer should be characterized as closely as possible in order to accurately predict the contact pressure distribu tions at the GDL interfaces. Improper estimations of the contact pressures may lead to incorrect values of the contact resistances, which may result in significant errors when modeling fuel cell performance using multiphysics models.

\subsection{Sensitivity analysis}

As a final step, in this section we present a parametric study to asses the influence of the geometrical parameters (rib width, $w_{\text {rib }}$, GDL thickness, $t_{G D L}$, and fillet radius) and the GDL mechanical properties (shear modulus, $G_{x y}$, and in plane Young's modulus, $E_{x} \quad E_{z}$ ) in the inhomogeneous compres sion phenomenon. The representative variables used to compare the results are the porosity field and the partial intrusion into the channel. In all cases, the parameter under study is varied above and below the reference value, which occupies the central position in the figures.

4.4.1. Geometric parameters: $\mathrm{w}_{\text {rib }}, \mathrm{t}_{\mathrm{GDL}}$, and fillet radius Fig. 9 shows the porosity field and GDL intrusion for three different rib widths: $w_{\text {rib }} 600,500$, and $400 \mu \mathrm{m}$. As the rib
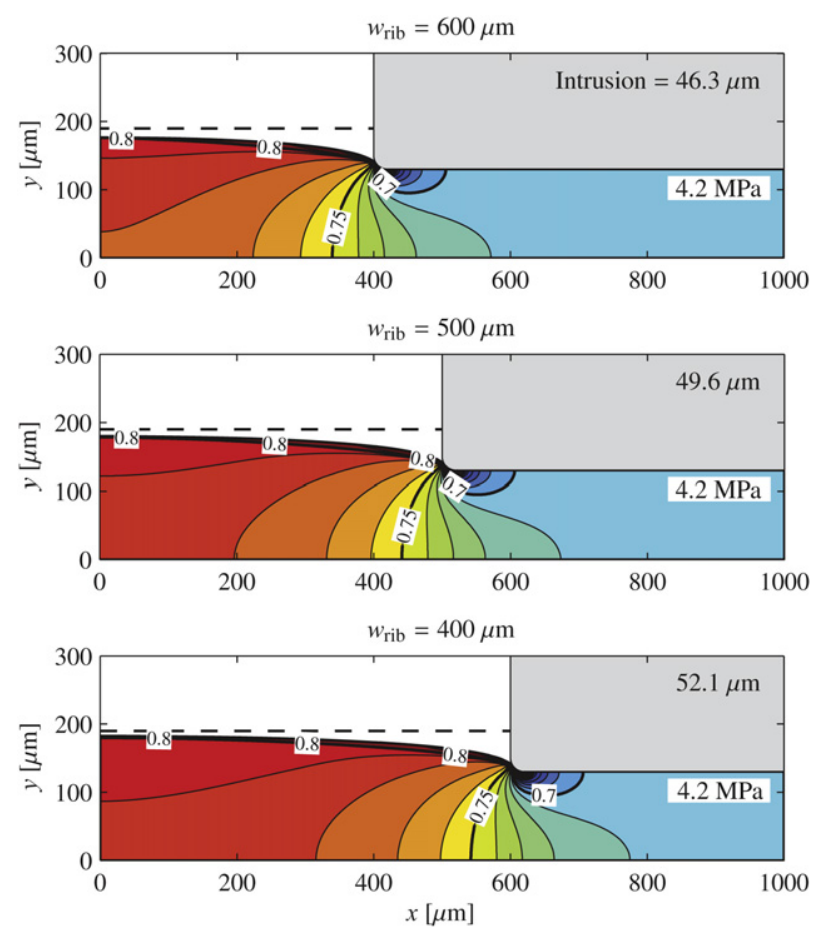

Fig. 9 - Porosity field $\phi(x, y)$, GDL intrusion into the channel, and compression stress at the rib symmetry plane obtained for different values of the rib width, $w_{\text {rib, }}$ corresponding to an imposed rib displacement of $60 \mu \mathrm{m}$ ( $32 \%$ of the initial GDL thickness, $\left.t_{\mathrm{GDL}}=190 \mu \mathrm{m}\right)$ and an initial porosity $\phi_{0}=0.8$. 
width decreases, the size of the low porosity region under the rib is seen to decrease correspondingly, while the GDL intru sion and the porosity under the channel increase because the rib compression effect is less effectively transmitted to this region. Although all these effects are beneficial in terms of reducing mass transport resistances, the use of exceedingly thin ribs would reduce the available area for the transport of electrons to the current collectors. As a consequence, an optimal rib width is expected to exist which maximizes fuel cell performance by a combination of low interfacial contact resistance and good porosity distribution for the GDL [42].

Fig. 10 shows the porosity field and GDL intrusion for three different initial GDL thicknesses, corresponding to Toray ${ }^{\circledR}$ carbon paper TGP H series: $t_{\mathrm{GDL}} 110 \mu \mathrm{m}$ (TGP H 030), $190 \mu \mathrm{m}$ (TGP H 060), and $280 \mu \mathrm{m}$ (TGP H 090). In this study, the imposed rib displacement was kept as a constant percentage (32\%) of the initial GDL thickness, as would result from the application of approximately the same compressive load in all cases. It is worth noting that as $t_{\mathrm{GDL}}$ decreases the transition fan like region between the channel and rib becomes more localized due to the higher concentration of rib edge effects. This results in higher porosities below the channel and, consequently, higher relative GDL intrusions (defined as the absolute intrusion over the initial GDL thickness).

Fig. 11 shows the porosity field and GDL intrusion for three different values of the fillet radius: 40,80 , and $160 \mu \mathrm{m}$, above the nominal $20 \mu \mathrm{m}$ considered in the rest of the paper. As can be seen, increasing the fillet radius reduces the rib-GDL contact surface as the first point of contact moves away from the
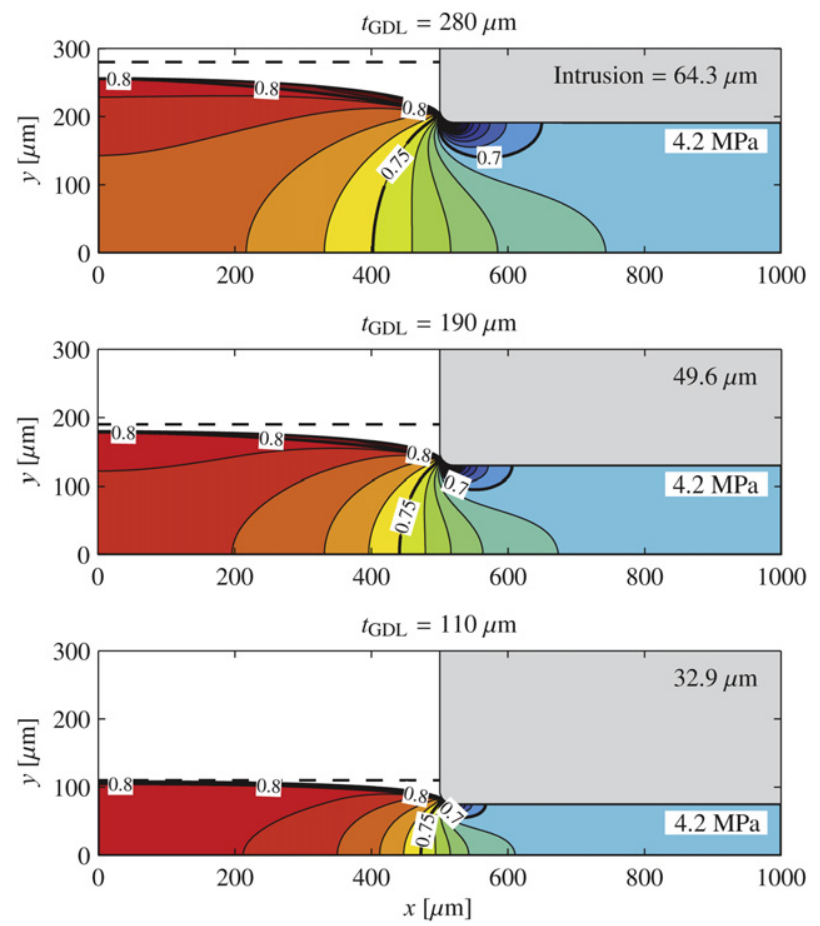

Fig. 10 - Porosity field $\phi(x, y)$, GDL intrusion into the channel, and compression stress at the rib symmetry plane obtained for different values of the initial GDL thickness, $t_{\mathrm{GDL}}$, corresponding to an imposed rib displacement of $60 \mu \mathrm{m}$ (32\% of the initial GDL thickness, $\left.t_{\mathrm{GDL}}=190 \mu \mathrm{m}\right)$ and an initial porosity $\phi_{0}=0.8$.
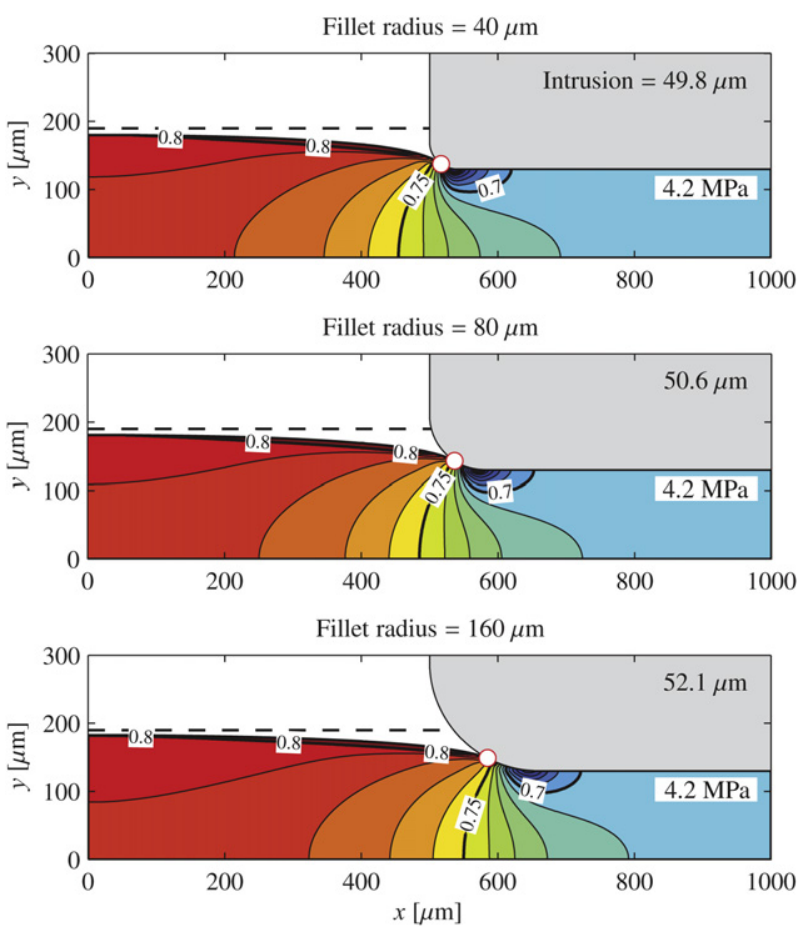

Fig. 11 - Porosity field $\phi(x, y)$, GDL intrusion into the channel, and compression stress at the rib symmetry plane obtained for different values of the fillet radius and for an imposed rib displacement of $60 \mu \mathrm{m}$ (32\% of the initial GDL thickness, $t_{G D L}=190 \mu \mathrm{m}$ ) and an initial porosity $\phi_{0}=0.8$. The hollow circle represents the first point of contact between the rib and the GDL.

channel, and also smoothes out the contact pressure peak that appears below the filleted rib corner, an effect not shown in the figure. Note that the reduction of the rib-GDL contact surface is qualitatively similar to that observed for smaller rib widths in Fig. 9, leading to a translation of the porosity field to the right by a distance of the order of the fillet radius without any signifi cant effect other than a slight increase in GDL intrusion.

\subsubsection{GDL mechanical properties: $\mathrm{G}_{\mathrm{xy}}$ and $\mathrm{E}_{\mathrm{x}} \quad \mathrm{E}_{\mathrm{z}}$}

The influence of the shear modulus $\left(G_{x y}\right)$ on the porosity field is qualitatively similar to that described above for the GDL thickness. As can be seen in Fig. 12, decreasing $G_{x y}$ reduces the size of the intermediate porosity region. Similarly, the intru sion is higher for smaller values of $G_{x y}$, as already noted by Kleemann et al. [54]. This behavior can be explained qualita tively in terms of the material shear stiffness: the lower the opposition of the material to deform by shear, the steeper the transition between the compressed (rib) and uncompressed (channel) regions, so that a larger region below the channel remains unaffected by the rib compression.

As observed in Fig. 13, the similarity in the effects of $G_{x y}$ and $t_{G D L}$ is not limited to the porosity field. Specifically, the interfacial contact pressure distributions resulting from different combinations of initial GDL thickness, $t_{G D L}$, and shear modulus, $G_{x y}$, turn also to be very similar, showing steeper variations of the contact pressure for smaller values of $t_{\mathrm{GDL}}$ and $G_{x y}$. 

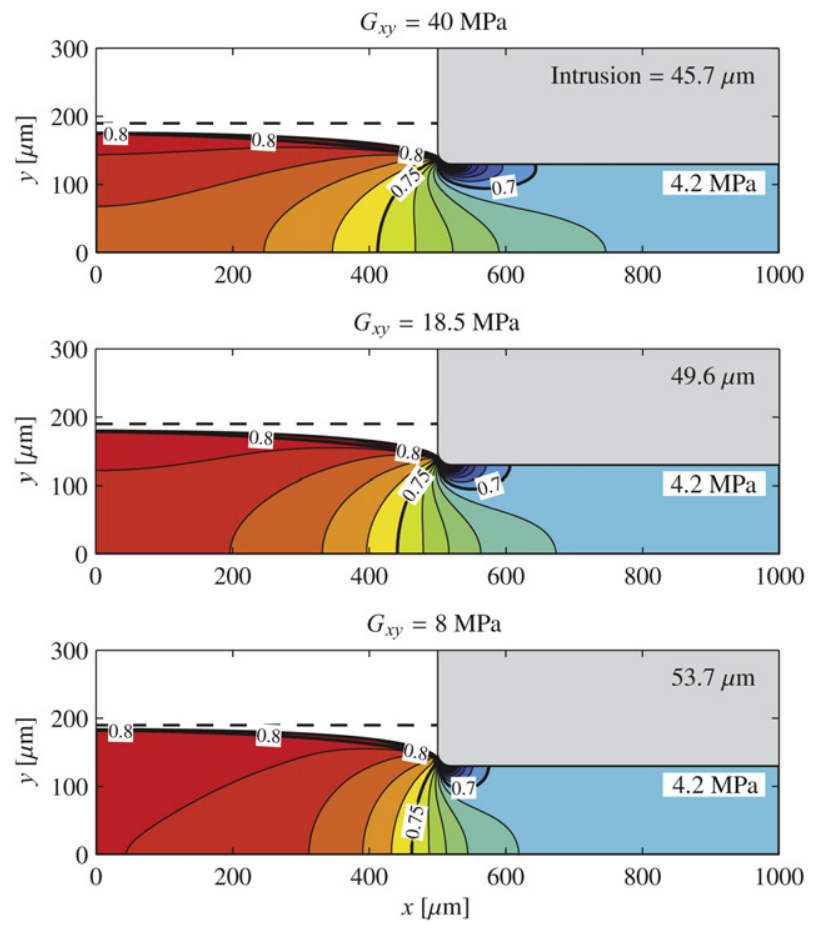

Fig. 12 - Porosity field $\phi(x, y)$, GDL intrusion into the channel, and compression stress at the rib symmetry plane obtained for different values of the shear modulus, $G_{x y}$, corresponding to an imposed rib displacement of $60 \mu \mathrm{m}\left(32 \%\right.$ of the initial GDL thickness, $\left.t_{\mathrm{GDL}}=190 \mu \mathrm{m}\right)$ and an initial porosity $\phi_{0}=0.8$.

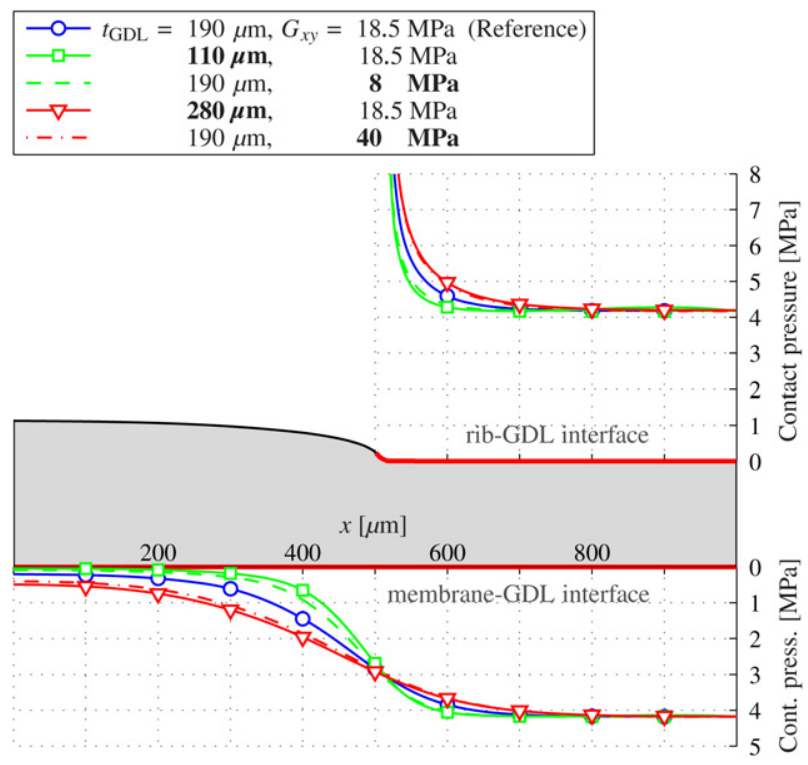

Fig. 13 - Contact pressure distribution at the rib-GDL (top) and membrane-GDL (bottom) interfaces obtained for different values of the initial GDL thickness, $t_{\mathrm{GDL}}$, and shear modulus, $G_{x y}$, corresponding to an imposed rib displacement of $60 \mu \mathrm{m}$ (32\% of the initial GDL thickness, $t_{\mathrm{GDL}}=190 \mu \mathrm{m}$ ) and an initial porosity $\phi_{0}=0.8$. Note the inverse vertical scale of the lower graph.

Table 4 - Sensitivity analysis in terms of relative GDL intrusion.

\begin{tabular}{lccc} 
Parameter & Value & Intrusion/ $\mathrm{t}_{\mathrm{GDL}}[\%]$ & Sensitivity [\%] \\
\hline$w_{\text {rib }}[\mu \mathrm{m}]$ & 600 & 24.4 & 33.3 \\
& 500 & 26.1 & \\
& 400 & 27.4 & 25.2 \\
$t_{\mathrm{GDL}}[\mu \mathrm{m}]$ & 280 & 23.0 & 25.4 \\
& 190 & 26.1 & 34.6 \\
$\mathrm{G}_{\text {xy }}[\mathrm{MPa}]$ & 110 & 29.9 & 6.8 \\
& 40 & 24.1 & \\
& 18.5 & 26.1 & 14.6 \\
$E_{x}=E_{y}[\mathrm{GPa}]$ & 5 & 28.3 & 0.4 \\
& 7 & 25.3 & 11.0 \\
\hline
\end{tabular}

The influence of the in plane Young's modulus $\left(E_{x} \quad E_{z}\right)$ in the numerical results is so small that it was necessary to change its value by an order of magnitude to find significant differences in the porosity distribution or the intrusion value (see Section 4.4.3 for details). Anyway, the simulations indicate again that when reducing the material in plane stiffness a larger region below the channel remains unaffected, resulting in slightly higher porosities and GDL intrusions in this region.

4.4.3. Sensitivity analysis in terms of relative GDL intrusion Table 4 summarizes the value of the relative intrusion, i.e. the ratio of the absolute intrusion to the initial GDL thickness, corresponding to the different combinations of geometric and mechanical parameters considered in the previous section. It also shows the sensitivity of the relative intrusion with respect to the different parameters, calculated as the ratio of the rela tive variation in the effect (the relative intrusion) to the relative variation in the cause (the considered parameter). All simula tions correspond to an imposed rib displacement of $32 \%$ of the initial GDL thickness.

The results show that both $w_{\text {rib }}$ and $t_{\mathrm{GDL}}$ have quantitatively similar effects when measured in terms of the relative intrusion, with a sensitivity of the order of $30 \%$ in both cases. Although less pronounced, the effect of $G_{x y}$ is still relevant, showing a sensi tivity of about $15 \%$ (7\%) for values of $G_{x y}$ smaller (larger) than the reference value. By contrast, the results show a modest effect of the in plane Young's modulus, with a sensitivity about $11 \%$ for significantly (i.e. tenfold) smaller values of $E_{x} \quad E_{z}$, and a virtually negligible sensitivity for values above the reference value (shown in boldface letters in the table).

As a final remark, it is interesting to note that the sensi tivity of the relative intrusion to the different parameters under study is always negative, indicating smaller relative intrusions for larger values of $w_{\mathrm{rib}}, t_{\mathrm{GDL}}, G_{x y}$, and $E_{x} \quad E_{z}$.

\section{Conclusions}

A FEM model has been developed to simulate the inhomoge neous assembly compression of PEM fuel cell gas diffusion layers. The model, validated with experimental measure ments and numerical results found in the literature, takes into account the nonlinear orthotropic properties of TGP H series 
Toray ${ }^{\circledR}$ carbon paper, a material commonly used as GDL in PEM fuel cells. The numerical simulations are expected to predict well the effects of the inhomogeneous compression of the porous layer corresponding to an imposed rib displace ment, including the porosity/diffusivity fields and the contact pressure distributions at the interfaces with the rib and the membrane. In all cases, the solutions exhibit a region of high compression and large porosity reduction under the rib, a region of low compression and almost unperturbed porosity under the channel, and a fan like transition region below the channel rib interface, which becomes more and more local ized for decreasing values of $t_{G D L}$ and $G_{x y}$.

As part of the study, we carried out a sensitivity analysis to characterize the effect of the different parameters involved in the model. This analysis showed that the geometrical parame ters $w_{\text {rib }}$ and $t_{\mathrm{GDL}}$ both have a major impact on the solution, while the mechanical parameters $E_{y}\left(\varepsilon_{y}\right), G_{x y}$ and $E_{x} \quad E_{z}$ exhibit different qualitative and quantitative effects. Thus, while the characterization of the nonlinear through plane Young's modulus $E_{y}\left(\varepsilon_{y}\right)$ is of primary importance for the determination of the maximum contact pressures, located under the rib, the shear modulus $G_{x y}$ is of greater relevance for the determination of the actual shape of the contact pressure distribution, with lower material shear stiffness resulting in steeper contact pressure distributions. By way of contrast, the extreme mechanical anisotropy of the material reduces the impact of the in plane Young's modulus $E_{x} \quad E_{z}$ (which is larger than $E_{y}\left(\varepsilon_{y}\right)$ and $G_{x y}$ by more than two orders of magnitude) in the results.

Among the main conclusions of the work, it is noteworthy that the linear isotropic models widely used in the literature tend to overestimate the porosity under the channel region and the partial intrusion of the GDL. In addition, they may predict either higher or lower contact pressures depending on the value of the through plane Young's modulus considered, and typically result in steeper contact pressure profiles.

Further extensions of the present model would be worth while exploring. Thus, the analysis may be extended to study the effect of a microporous layer, or to account for the hyster etic behavior of the GDL, as long as the required data were available. The model also provides a detailed description of the transmission of mechanical stresses from rib to membrane, which would be useful in the study of the mechanical loads suffered by the membrane during fuel cell assembly and operation. Of particular interest would be the study of the mechanical failure of MEA components near the edges of the cell $[79,80]$. This analysis would require an appropriate consti tutive model for the membrane (including both swelling and elastic-viscoplastic effects [81,82]) and should include the restraining effect of the sealing gasket [46]. The porosity/ diffusivity fields and contact pressure distributions reported in this paper constitute also a necessary first step towards the multiphysics modeling of the effects of inhomogeneous GDL compression on overall fuel cell performance.

\section{Acknowledgements}

This work was supported by Project ENE2008 06683 C03 02 of the Spanish Ministerio de Ciencia e Innovación (GS1).
[1] EG\&G Technical Services Inc. Fuel cell handbook. 7th ed. U.S. Dept. of Energy, Office of Fossil Energy, Morgantown, WV, USA; 2004

[2] Barbir F. PEM fuel cells: theory and practice. Elsevier Academic Press; 2005.

[3] Mehta V, Cooper JS. Review and analysis of PEM fuel cell design and manufacturing. J Power Sources 2003;114:32 53

[4] Li X, Sabir I. Review of bipolar plates in PEM fuel cells: flow field designs. Int J Hydrogen Energy 2005;30:359 71.

[5] Hermann A, Chaudhuri T, Spagnol P. Bipolar plates for PEM fuel cells: a review. Int J Hydrogen Energy 2005;30: 1297302.

[6] Cindrella L, Kannan AM, Lin JF, Saminathan K, Ho Y, Lin CW, et al. Gas diffusion layer for proton exchange membrane fuel cells a review. J Power Sources 2009;194:146 60.

[7] Radhakrishnan V, Haridoss P. Differences in structure and property of carbon paper and carbon cloth diffusion media and their impact on proton exchange membrane fuel cell flow field design. Mater Des 2011;32:861 8.

[8] Escribano S, Blachot J, Ethève J, Morin A, Mosdale R. Characterization of PEMFCs gas diffusion layers properties. J Power Sources 2006;156:8 13.

[9] Ge J, Higier A, Liu H. Effect of gas diffusion layer compression on PEM fuel cell performance, J. Power Sources 2006;159: 9227.

[10] Gostick JT, Fowler MW, Pritzker MD, Ioannidis MA, Behra LM. In plane and through plane gas permeability of carbon fiber electrode backing layers. J Power Sources 2006;162:228 38.

[11] Feser JP, Prasad AK, Advani SG. Experimental characterization of in plane permeability of gas diffusion layers. J Power Sources 2006;162:1226 31.

[12] Zhou Y, Lin G, Shih AJ, Hu SJ. A micro scale model for predicting contact resistance between bipolar plate and gas diffusion layer in PEM fuel cells. J Power Sources 2007;163: 77783.

[13] Chang WR, Hwang JJ, Weng FB, Chan SH. Effect of clamping pressure on the performance of a PEM fuel cell. J Power Sources 2007;166:149 54.

[14] Lin J H, Chen W H, Su Y J, Ko T H. Effect of gas diffusion layer compression on the performance in a proton exchange membrane fuel cell. Fuel 2008;87:2420 4.

[15] Yim S D, Kim B J, Sohn Y J, Yoon Y G, Park G G, Lee W Y, et al. The influence of stack clamping pressure on the performance of PEM fuel cell stack. Curr Appl Phys 2010;10: S59 61.

[16] Radhakrishnan V, Haridoss P. Effect of cyclic compression on structure and properties of a Gas Diffusion Layer used in PEM fuel cells. Int J Hydrogen Energy 2010;35:11107 18.

[17] Zhu Y, Liu C, Liang J, Wang L. Investigation of the effects of compression pressure on direct methanol fuel cell. J Power Sources 2011;196:264 9.

[18] Kusoglu A, Karlsson AM, Santare MH, Cleghorn S, Johnson WB. Mechanical response of fuel cell membranes subjected to a hygro thermal cycle. J Power Sources 2006;161: 98796.

[19] Kusoglu A, Karlsson AM, Santare MH, Cleghorn S, Johnson WB. Mechanical behavior of fuel cell membranes under humidity cycles and effect of swelling anisotropy on the fatigue stresses. J Power Sources 2007;170:345 58.

[20] Zhou Y, Lin G, Shih AJ, Hu SJ. Assembly pressure and membrane swelling in PEM fuel cells. J Power Sources 2009; 192:544 51.

[21] Silberstein MN, Boyce MC. Hygro thermal mechanical behavior of Nafion during constrained swelling. J Power Sources 2011;196:3452 60 . 
[22] Solasi R, Zou Y, Huang X, Reifsnider K, Condit D. On mechanical behavior and in plane modeling of constrained PEM fuel cell membranes subjected to hydration and temperature cycles. J Power Sources 2007;167:366 77.

[23] Kusoglu A, Santare MH, Karlsson AM, Cleghorn S, Johnson WB. Numerical investigation of mechanical durability in polymer electrolyte membrane fuel cells. J Electrochem Soc 2010;157:B705 13.

[24] Lu Z, Kim C, Karlsson AM, Cross III JC, Santare MH. Effect of gas diffusion layer modulus and land groove geometry on membrane stresses in proton exchange membrane fuel cells. J Power Sources 2011;196:4646 54.

[25] Mishra V, Yang F, Pitchumani R. Measurement and prediction of electrical contact resistance between gas diffusion layers and bipolar plate for applications to PEM fuel cells. J Fuel Cell Sci Technol 2004;1:2 9.

[26] Nitta I, Himanen O, Mikkola M. Contact resistance between gas diffusion layer and catalyst layer of PEM fuel cell. Electrochem Commun 2008;10:47 51.

[27] Nitta I, Hottinen T, Mikkola M. Thermal conductivity and contact resistance of compressedgas diffusion layer of PEM fuel cell. Fuel Cells 2008;8:111 9.

[28] Nitta I, Hottinen T, Himanen O, Mikkola M. Inhomogeneous compression of PEMFC gas diffusion layer: part I. Experimental. J Power Sources 2007;171:26 36.

[29] Hottinen T, Himanen O, Karvonen S, Nitta I. Inhomogeneous compression of PEMFC gas diffusion layer: part II. Modeling the effect. J Power Sources 2007;171:113 21.

[30] Nitta I. Inhomogeneous compression of PEMFC gas diffusion layers, Doctoral dissertation, Helsinki (Finland); 2008.

[31] Bazylak A, Sinton D, Liu Z S, Djilali N. Effect of compression on liquid water transport and microstructure of PEMFC gas diffusion layers. J Power Sources 2007;163:784 92.

[32] Yoon W, Huang X. A multiphysics model of PEM fuel cell incorporating the cell compression effects. J Electrochem Soc 2010;157:B680 90.

[33] Lee W, Ho C H, Van Zee JW, Murthy M. The effects of compression and gas diffusion layers on the performance of a PEM fuel cell. J Power Sources 1999;84:45 51.

[34] Mathias M, Roth J, Fleming J, Lehnert W. Chapter 46. In: Vielstich W, Gasteiger HA, Lamm A, editors. Handbook of fuel cells fundamentals, technology and applications. Fuel cell technology and applications, vol. 3. John Wiley \& Sons, Ltd; 2003.

[35] Lee S J, Hsu C D, Huang C H. Analyses of the fuel cell stack assembly pressure. J Power Sources 2005;145:353 61 .

[36] Wang C Y. Fundamental models for fuel cell engineering. Chem Rev 2004;104:4727 66.

[37] Chu H S, Yeh C, Chen F. Effects of porosity change of gas diffuser on performance of proton exchange membrane fuel cell. J Power Sources 2003;123:1 9.

[38] Roshandel R, Farhanieh B, Saievar Iranizad E. The effects of porosity distribution variation on PEM fuel cell performance. Renew Energy 2005;30:1557 72.

[39] Roshandel R, Farhanieh B. The effects of non uniform distribution of catalyst loading on polymer electrolyte membrane fuel cell performance. Int J Hydrogen Energy 2007;32:4424 37.

[40] Sui PC, Djilali N. Analysis of coupled electron and mass transport in the gas diffusion layer of a PEM fuel cell. J Power Sources 2006;161:294 300.

[41] Su ZY, Liu CT, Chang HP, Li CH, Huang KJ, Sui PC. A numerical investigation of the effects of compression force on PEM fuel cell performance. J Power Sources 2008;183: 18292.

[42] Zhou P, Wu CW, Ma GJ. Contact resistance prediction and structure optimization of bipolar plates. J Power Sources 2006;159:1115 22.
[43] Zhou P, Wu CW, Ma GJ. Influence of clamping force on the performance of PEMFCs. J Power Sources 2007;163:874 81.

[44] Zhou P, Wu CW. Numerical study on the compression effect of gas diffusion layer on PEMFC performance. J Power Sources 2007;170:93 100.

[45] Kandlikar SG, Lu Z, Lin TY, Cooke D, Daino M. Uneven gas diffusion layer intrusion in gas channel arrays of proton exchange membrane fuel cell and its effects on flow distribution. J Power Sources 2009;194:328 37.

[46] Bograchev D, Gueguen M, Grandidier J, Martemianov S. Stress and plastic deformation of MEA in fuel cells stresses generated during cell assembly. J Power Sources 2008;180: 393401.

[47] Bograchev D, Gueguen M, Grandidier J, Martemianov S. Stress and plastic deformation of MEA in running fuel cell. Int J Hydrogen Energy 2008;33:5703 17.

[48] Lai X, Liu D, Peng L, Ni J. A mechanical electrical finite element method model for predicting contact resistance between bipolar plate and gas diffusion layer in PEM fuel cells. J Power Sources 2008;182:153 9.

[49] Liu D, Peng L, Lai X. Effect of dimensional error of metallic bipolar plate on the GDL pressure distribution in the PEM fuel cell. Int J Hydrogen Energy 2009;34:990 7.

[50] Zhang L, Liu Y, Song H, Wang S, Zhou Y, Jack Hu S. Estimation of contact resistance in proton exchange membrane fuel cells. J Power Sources 2006;162:1165 71.

[51] Akiki T, Charon W, Iltchev M, Accary G, Kouta R. Influence of local porosity and local permeability on the performance of a polymer electrolyte membrane fuel cell. J Power Sources 2010;195:5258 68.

[52] Taymaz I, Benli M. Numerical study of assembly pressure effect on the performance of proton exchange membrane fuel cell. Energy 2010;35:2134 40.

[53] Lai Y H, Rapaport PA, Ji C, Kumar V. Channel intrusion of gas diffusion media and the effect on fuel cell performance. J Power Sources 2008;184:120 8.

[54] Kleemann J, Finsterwalder F, Tillmetz W. Characterisation of mechanical behaviour and coupled electrical properties of polymer electrolyte membrane fuel cell gas diffusion layers. J Power Sources 2009;190:92 102.

[55] Serincan MF, Pasaogullari U. Effect of gas diffusion layer anisotropy on mechanical stresses in a polymer electrolyte membrane. J Power Sources 2011;196:1314 20.

[56] Serincan MF, Pasaogullari U. Mechanical behavior of the membrane during the polymer electrolyte fuel cell operation. J Power Sources 2011;196:1303 13.

[57] Pharoah JG, Karan K, Sun W. On effective transport coefficients in PEM fuel cell electrodes: anisotropy of the porous transport layers. J Power Sources 2006;161:214 24.

[58] HKS. Abaqus v6.4 user's manual, version 6.4 edition. Richmond, USA: ABAQUS Inc.; 2003.

[59] Brodrecht D, Liu S, Djilali N. Finite element analysis of a fuel cell membrane electrode assembly under compression. Proceedings of international green energy conference (IGEC 1), Waterloo (Canada); 2005.

[60] Li Y. Experimental studies on the mechanical durability of proton exchange membranes. Doctoral dissertation, Blacksburg, VA (USA); 2008.

[61] Lai YH, Li Y, Rock JA. A novel full field experimental method to measure the local compressibility of gas diffusion media. J Power Sources 2010;195:3215 23.

[62] Sadeghi E, Djilali N, Bahrami M. Effective thermal conductivity and thermal contact resistance of gas diffusion layers in proton exchange membrane fuel cells. Part 2: hysteresis effect under cyclic compressive load. J Power Sources 2010;195:8104 9.

[63] Fu YB, Ogden RW. Nonlinear elasticity: theory and applications. London: Cambridge University Press; 2001. 
[64] Zhang XF, Andrieux F, Sun DZ. Pseudo elastic description of polymeric foams at finite deformation with stress softening and residual strain effects. Mater Des 2011;32:877 84.

[65] Suvorov AP, Elter J, Staudt R, Hamm R, Tudryn GJ, Schadler L, et al. Stress relaxation of PBI based membrane electrode assemblies. Int J Solids Structures 2008;45:5987 6000 .

[66] Mann RW, Baum GA, Habeger CC. Determination of all nine orthotropic elastic constants for machine made paper. Tappi J 1980;63:163 6.

[67] Delincé M, Delannay F. Elastic anisotropy of a transversely isotropic random network of interconnected fibres: non triangulated network model. Acta Mater 2004;52:1013 22.

[68] Matsuura T, Kato M, Hori M. Study on metallic bipolar plate for proton exchange membrane fuel cell. J Power Sources 2006;161:74 8.

[69] Yi P, Peng L, Lai X. A numerical model for predicting gas diffusion layer failure in proton exchange membrane fuel cells. J Fuel Cell Sci Technol 2011;8:011011.

[70] Toray carbon fiber paper "TGP H" datasheet, Toray Industries, Inc.

[71] Flückiger R. Transport phenomena on the channel rib scale of polymer electrolyte fuel cells. Doctoral dissertation, ETH Zurich (Switzerland); 2009.

[72] Flückiger R, Freunberger SA, Kramer D, Wokaun A, Scherer GG, Büchi FN. Anisotropic, effective diffusivity of porous gas diffusion layer materials for PEFC. Electrochim Acta 2008;54:551 9.

[73] Möst M, Rzepka M, Stimming U. Analysis of the diffusive mass transport in the anode side porous backing layer of a direct methanol fuel cell. J Power Sources 2009;191:456 64

[74] Kramer D, Freunberger SA, Flückiger R, Schneider IA, Wokaun A, Büchi FN, et al. Electrochemical diffusimetry of fuel cell gas diffusion layers. J Electroanal Chem 2008;612:63 77.

[75] Bruggeman DAG. Berechnung verschiedener physikalischer Konstanten von heterogenen Substanzen. Annalen der Physik, 5. Folge, Band 1935;24.

[76] Tomadakis MM, Sotirchos SV. Ordinary and transition regime diffusion in random fiber structures. AIChE J 1993;39: 397412.

[77] Becker J, Flückiger R, Reum M, Büchi FN, Marone F, Stampanonic M. Determination of material properties of gas diffusion layers: experiments and simulations using phase contrast tomographic microscopy. J Electrochem Soc 2009; 156:B1175 81.

[78] Das PK, Li X, Liu Z S. Effective transport coefficients in PEM fuel cell catalyst and gas diffusion layers: beyond Bruggeman approximation. Appl Energy 2010;87:2785 96.
[79] Huang X, Reifsnider K. In: Wang C Y, Pasaogullari U, editors. Durability of PEM fuel cell membranes. Modern aspects of electrochemistry 49: modeling and diagnostics of polymer electrolyte fuel cells; 2010. p. 143.

[80] Solasi R, Huang X, Reifsnider K. Creep and stress rupture of Nafion membranes under controlled environment. Mech Mater 2010;42:678 85.

[81] Solasi R, Zou Y, Huang X. A time and hydratation dependent viscoplastic model for polyelectrolyte membranes in fuel cells. Mech Time Depend Mater 2008;12:15 30.

[82] Silberstein MN, Boyce MC. Constitutive modeling of the rate, temperature, and hydration dependent deformation response of Nafion to monotonic and cyclic loading. J Power Sources 2010;195:5692 706 .

\section{Nomenclature}

Symbols

$D_{i, j}$ : Binary diffusion coefficient

E: Young's modulus

G: Shear modulus

t: Thickness

$\mathrm{u}$ : Displacement

V: Volume

w: Half width

$\mathrm{x}$ : Transverse coordinate in the material plane

y: Through plane coordinate

z: Longitudinal coordinate in the material plane

Greek letters

$\gamma$ : Shear strain

$\varepsilon$ : Longitudinal true strain

$v$ : Poisson's ratio

$\sigma$ : Normal true stress

$\tau_{\mathrm{xy}}$ : Shear stress

$\tau$ : Tortuosity

$\phi$ : Porosity

Subscripts and superscripts

0 : Initial

eff: Effective

ip: In plane

$p$ : Porous

tp: Through plane

V: Volumetric 